\title{
2-Deoxyglucose and sorafenib synergistically suppress the proliferation and motility of hepatocellular carcinoma cells
}

\author{
MINORU TOMIZAWA ${ }^{1}$, FUMINOBU SHINOZAKI ${ }^{2}$, YASUFUMI MOTOYOSHI ${ }^{3}$, \\ TAKAO SUGIYAMA $^{4}$, SHIGENORI YAMAMOTO ${ }^{5}$ and NAOKI ISHIGE ${ }^{6}$ \\ Departments of ${ }^{1}$ Gastroenterology, ${ }^{2}$ Radiology, ${ }^{3}$ Neurology, ${ }^{4}$ Rheumatology, ${ }^{5}$ Pediatrics and ${ }^{6}$ Neurosurgery, \\ National Hospital Organization, Shimoshizu Hospital, Yotsukaido, Chiba 284-0003, Japan
}

Received October 2, 2015; Accepted October 18, 2016

DOI: $10.3892 / \mathrm{ol} .2016 .5510$

\begin{abstract}
Cancer cells consume more glucose than normal cells, mainly due to their increased rate of glycolysis. 2-Deoxy-d-glucose (2DG) is an analogue of glucose, and sorafenib is a kinase inhibitor and molecular agent used to treat hepatocellular carcinoma (HCC). The present study aimed to demonstrate whether combining 2DG and sorafenib suppresses tumor cell proliferation and motility more effectively than either drug alone. HLF and PLC/PRF/5 HCC cells were incubated with sorafenib with or without $1 \mu \mathrm{M}$ $2 \mathrm{DG}$, and subjected to a proliferation assay. A scratch assay was then performed to analyze cell motility following the addition of 2DG and sorafenib in combination, and each agent alone. RNA was isolated and subjected to reverse transcription-quantitative polymerase chain reaction to analyze the expression of cyclin D1 and matrix metalloproteinase-9 (MMP9) following the addition of 2DG and sorafenib in combination and each agent alone. Proliferation was markedly suppressed in cells cultured with $1 \mu \mathrm{M} 2 \mathrm{DG}$ and $30 \mu \mathrm{M}$ sorafenib compared with cells cultured with either agent alone $(\mathrm{P}<0.05)$. In addition, levels of Cyclin D1 expression decreased in cells exposed to $3 \mu \mathrm{M}$ sorafenib and $1 \mu \mathrm{M} 2 \mathrm{DG}$ compared with cells exposed to $2 \mathrm{DG}$ or sorafenib alone $(\mathrm{P}<0.05)$. Scratch assay demonstrated that the distance between the growing edge of the cell sheet and the scratched line was shorter in cells cultured with sorafenib and 2DG than in cells cultured with $2 \mathrm{DG}$ or sorafenib alone $(\mathrm{P}<0.05)$. Levels of MMP9 expression decreased more in cells treated with both sorafenib and 2DG than in cells treated with 2DG or sorafenib alone $(\mathrm{P}<0.05)$. Therefore, $2 \mathrm{DG}$ and sorafenib in combination suppressed the proliferation and motility of HCC cells more effectively than 2DG or sorafenib alone, and
\end{abstract}

Correspondence to: Dr Minoru Tomizawa, Department of Gastroenterology, National Hospital Organization Shimoshizu Hospital, 934-5 Shikawatashi, Yotsukaido, Chiba 284-0003, Japan E-mail: nihminor-cib@umin.ac.jp

Key words: 2-Deoxyglucose, sorafenib, HCC, cyclin D1, matrix metalloproteinase a cancer treatment combining both drugs may be more effective than sorafenib alone.

\section{Introduction}

Hepatocellular carcinoma (HCC) originates in the liver following long-term infection with the hepatitis $\mathrm{B}$ or $\mathrm{C}$ virus (1), and recent research has linked non-alcoholic steatohepatitis to HCC etiology (2). Current treatments include resection, local ablation, transcatheter arterial chemoembolization, targeted chemotherapy, and palliative care $(3,4)$. In addition, sorafenib, a kinase inhibitor, is increasingly used to treat HCC (5), however there are problems associated with its use, most notably, the ability of HCC cells to develop resistance to it $(6,7)$.

Cancer cells depend on glycolysis for energy production and survival, and they exhibit significantly higher rates of glycolysis than healthy tissues even when oxygen is plentiful, a phenomenon known as the Warburg effect (8). As they require more glucose than healthy tissue and metabolise this glucose primarily by glycolysis, inhibiting glycolysis may be a more effective way of eradicating cancer cells (9), and consequently be developed as a treatment for cancer (10).

2-Deoxy-d-glucose (2DG) is an analogue of glucose which cannot undergo glycolysis (11). Cancer cells take up 2DG in a similar way to glucose, however, they cannot metabolize it to produce energy. Therefore energy production is impaired, and cancer cells are damaged (11).

The safety of 2DG in humans has been established through its application in positron emission tomography (12). If the combination of sorafenib and 2DG exert significantly stronger anti-tumor effects than either drug alone, it may provide a novel therapeutic strategy to treat HCC.

The present study sought to investigate the suppressive effects of sorafenib and 2DG in combination on the proliferation and motility of HCC cells compared with sorafenib alone.

\section{Materials and methods}

Cells and cell culture. Human hepatocellula carcinoma cells, HLF cells and PLC/PRF/5 cells were purchased from the RIKEN BioResource Centre Cell Bank (Tsukuba, Japan), and 
cultured in Dulbecco's modified Eagle's medium (DMEM; Sigma-Aldrich, St. Louis, MO, USA) supplemented with $10 \%$ fetal bovine serum (FBS; Thermo Fisher Scientific, Inc., Waltham, MA, USA). The cells were cultured in $10 \mathrm{~cm}$ dishes (Asahi Techno Glass, Yoshida-cho, Japan) with 5\% carbon dioxide at $37^{\circ} \mathrm{C}$ in a humidified chamber.

Cell proliferation assay. Cells were trypsinized, harvested, and split onto 96-well plates (Asahi Techno Glass) at a density of 1,000 cells/well. Varying concentrations of sorafenib (JS Research Chemicals Trading, Wedel, Germany), 0, 1, 3, 10, and $30 \mu \mathrm{M}$, were added to the media, and either 0 or $1 \mu \mathrm{M}$ 2DG (Sigma-Aldrich) was added. Following $72 \mathrm{~h}$ culture, the cells were subjected to an MTS assay, (Promega Corporation, Madison, WI, USA) according to the manufacturer instructions. During the assay, the cells reduce MTS to a colored formazan product with an absorbance maximum at $490 \mathrm{~nm}$. The absorbance of the product was subsequently measured using an iMark Microplate absorbance reader (Bio-Rad Laboratories Inc., Hercules, CA, USA).

Scratch assay. HLF and PLC/PRF/5 cells were plated on 4-well chamber slides (BD Biosciences, Franklin Lakes, NJ, USA), and scratched with a sterile razor when they reached confluence. Immediately after scratch, the cells were incubated for $48 \mathrm{~h}$ with $1 \mu \mathrm{M}$ sorafenib, $3 \mu \mathrm{M} 2 \mathrm{DG}$, or a combination of both. Following incubation, they were stained with hematoxylin and eosin. The slides were observed under an AX80 microscope (Olympus Corporation, Tokyo, Japan, magnification $\mathrm{x} 100$ ), and the distance between the scratched line and the growing edges of the cells was measured at five different points.

Reverse transcription-quantitative polymerase chain reaction $(R T-q P C R)$. Cells were cultured in 6-well plates (Asahi Techno Glass), and when they reached $70 \%$ confluence, were incubated with $1 \mu \mathrm{M}$ sorafenib, $3 \mu \mathrm{M} 2 \mathrm{DG}$, or a combination of both. Following 48-h culture, RNA was isolated with Isogen (Nippon Gene Co., Tokyo, Japan) according to the manufacturer's instructions. The isolated RNA was suspended in autoclaved Tris-EDTA buffer (pH 8.0). Total RNA $(5 \mu \mathrm{M})$ was subjected to cDNA synthesis with SuperScript III and oligo(dT) following manufacturer instructions (Thermo Fisher Scientific). RT-qPCR was performed using Fast SYBR Green Master Mix (Thermo Fisher Scientific) with Mini Opticon (Bio-Rad). RT-qPCR was performed for 40 cycles, with $5 \mathrm{sec}$ of denaturation and $5 \mathrm{sec}$ of annealing-extension. Primer sequences are presented in Table I. RPL19, a housekeeping gene that is constitutively expressed (13), was used as an internal control. The expression levels of gene were analyzed automatically by the Mini Opticon system (Bio-Rad) based on delta-delta cycle threshold $(\mathrm{ddCt})$ method. Relative expression level of a gene was calculated as expression level of a gene divided by expression level of RPL19.

Statistical analysis. One-way analysis of variance (ANOVA) was used for statistical analysis with JMP software ver. 10.0.2 (SAS Institute, Cary, NC, USA). $\mathrm{P}<0.05$ was considered to indicate a statistically significant difference.

\section{Results}

To investigate whether 2DG enhanced the anti-proliferative effects of sorafenib, HLF cells (Fig. 1A) and PLC/PRF/5 cells (Fig. 1B) were cultured with $0,1,3,10$, and $30 \mu \mathrm{M}$ sorafenib either alone (black bars) or with 2DG (white bars). The combination of $1 \mu \mathrm{M} 2 \mathrm{DG}$ and $30 \mu \mathrm{M}$ sorafenib markedly suppressed cell proliferation of both cell types compared to sorafenib alone $(\mathrm{P}<0.05)$. This suggests that $2 \mathrm{DG}$ is able able to enhance the anti-proliferative effects of sorafenib in HLF and PLC/PRF/5 cells.

To clarify the effect of sorafenib and 2DG on the cell cycle, levels of cyclin D1 expression were analyzed by RT-qPCR in HLF cells (Fig. 2A) and PLC/PRF/5 cells (Fig. 2B). The expression of cyclin D1 in both cell types decreased more in cells exposed to sorafenib and 2DG compared with cells exposed to $2 \mathrm{DG}$ or sorafenib alone ( $\mathrm{P}<0.05$; Fig. 2). This demonstrates that sorafenib and 2DG together decrease cyclin D1 expression more effectively than either drug alone.

To investigate the effects of sorafenib and 2DG on cell motility, a scratch assay was performed on HLF cells (Fig. 3A-D) and PLC/PRF/5 cells (Fig. 3E-H). All cells were cultured in 4-well chamber slides, and following scratching with a sterile razor, $3 \mu \mathrm{M}$ sorafenib (Fig. 3B and F), $1 \mu \mathrm{M} 2 \mathrm{DG}$ (Fig. 3C and G), or a combination of both (Fig. 3D and H) were added. Cells were then stained, and the distance between the growing edge of the cell sheet and the scratched line was measured in HLF cells (Fig. 3I) and PLC/PRF/5 cells (Fig. 3J). The distance was shorter in cells cultured with both sorafenib and 2DG than in cells cultured with 2DG or sorafenib alone $(\mathrm{P}<0.05$; Fig. 3I and $\mathrm{J})$, suggesting that $2 \mathrm{DG}$ enhances the suppressive effects of sorafenib on cell motility.

Matrix metalloproteinase 9 (MMP9) is involved in cell motility and is overexpressed in HCC $(14,15)$. MMP9 expression was analyzed using RT-qPCR in HLF cells (Fig. 4A) and PLC/PRF/5 cells (Fig. 4B), and was significantly lower in cells cultured with a combination of sorafenib and 2DG than in cells cultured with either drug alone $(\mathrm{P}<0.05)$.

\section{Discussion}

Previous studies have demonstrated that 2-[(3-Carboxy1-oxoprogy1) amino]-2-deoxy-D-glucose, another analogue of glucose similar to $2 \mathrm{DG}$, suppresses the proliferation of cells from the human HCC HepG2 cell line (16). In the present study, $3 \mu \mathrm{M} 2 \mathrm{DG}$ and $30 \mu \mathrm{M}$ sorafenib significantly suppressed the proliferation of HLF and HCC PLC/PRF/5 cells. The difference in suppression of proliferation was significant at sorafenib concentrations $>10 \mu \mathrm{M}$.

Sorafenib and 2DG independently decrease cyclin D1 expression (Fig. 2), therefore it was expected that the combination of sorafenib and $2 \mathrm{DG}$ would synergistically suppress HCC proliferation at lower concentrations of sorafenib. This was observed in both HLF and PLC/PRF/5 cells. However, the reasons underlying the suppressive effects of $2 \mathrm{DG}$ and sorafenib combination on HCC cell proliferation remain unclear.

Metastasis is a result of HCC cell invasion from the primary site to local or distant tissue (17). The combination of 2DG and sorafenib suppressed cell motility more effectively than 2DG or sorafenib alone (Fig. 3). Additionally, 
Table I. Primers for real-time quantitative PCR.

\begin{tabular}{|c|c|c|c|c|c|c|}
\hline Name & Sequence & Description & $\begin{array}{l}\text { Product } \\
\text { size, bp }\end{array}$ & $\begin{array}{l}\text { Annealing } \\
\text { temp, }{ }^{\circ} \mathrm{C}\end{array}$ & Cycle & GenBank \\
\hline OMC355 & 5'-AGAGGCGGAGGAGAACAAACAG-3' & Cyclin D1 (F) & 180 & 60 & 40 & NM_053056 \\
\hline OMC356 & 5'-AGGCGGTAGTAGGACAGGAAGTTG-3' & Cyclin D1 (R) & & & & \\
\hline OMC749 & 5'-CCTGGGCAGATTCCAAACCT-3' & MMP9 (F) & 89 & 60 & 40 & NM_004994 \\
\hline OMC750 & 5'-GCAAGTCTTCCGAGTAGTTTTGGAT-3 & MMP9 (R) & & & & \\
\hline OMC321 & 5'-CGAATGCCAGAGAAGGTCAC-3' & RPL19 (F) & 157 & 60 & 40 & BC095445 \\
\hline OMC322 & 5'-CCATGAGAATCCGCTTGTTT-3' & RPL19 (R) & & & & \\
\hline
\end{tabular}

F, forward; $R$, reverse.
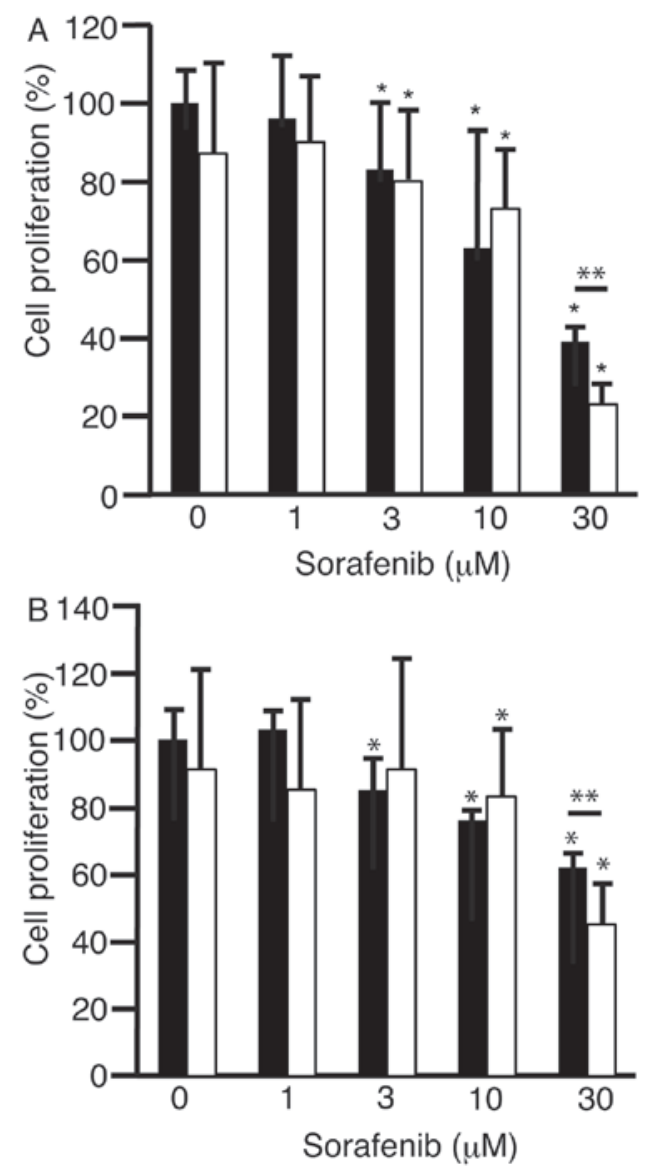

Figure 1.Cell proliferation assay. HLF cells (A) and PLC/PRF/5 cells (B) were added to $0,1,3,10$, and $30 \mu \mathrm{M}$ sorafenib either with (white bar), or without (black bar) $1 \mu \mathrm{M}$ 2DG. The cells were subjected to MTS assay to measure cell proliferation. ${ }^{*} \mathrm{P}<0.05$ against $0 \mu \mathrm{M} 2 \mathrm{DG}$ and sorafenib, ${ }^{* *} \mathrm{P}<0.05$ against $0 \mu \mathrm{M} 2 \mathrm{DG}$ and $30 \mu \mathrm{M}$ sorafenib. HLF, Human liver fibroblasts; PLC/PRF/5, the human PLC/PRF/5 hepatoma carcinoma cell line; 2DG, 2-deoxy-glucose.

expression of MMP9 significantly decreased when cells were treated with a combination of 2DG and sorafenib compared with 2DG or sorafenib alone (Fig. 4), suggesting that treatment with both 2DG and sorafenib may effectively inhibit metastasis.

2DG enhances the anti-tumor effects of other chemotherapeutic agents (18). In vivo studies have demonstrated that sections of tumors are hypoxic (19), and cancer cells are

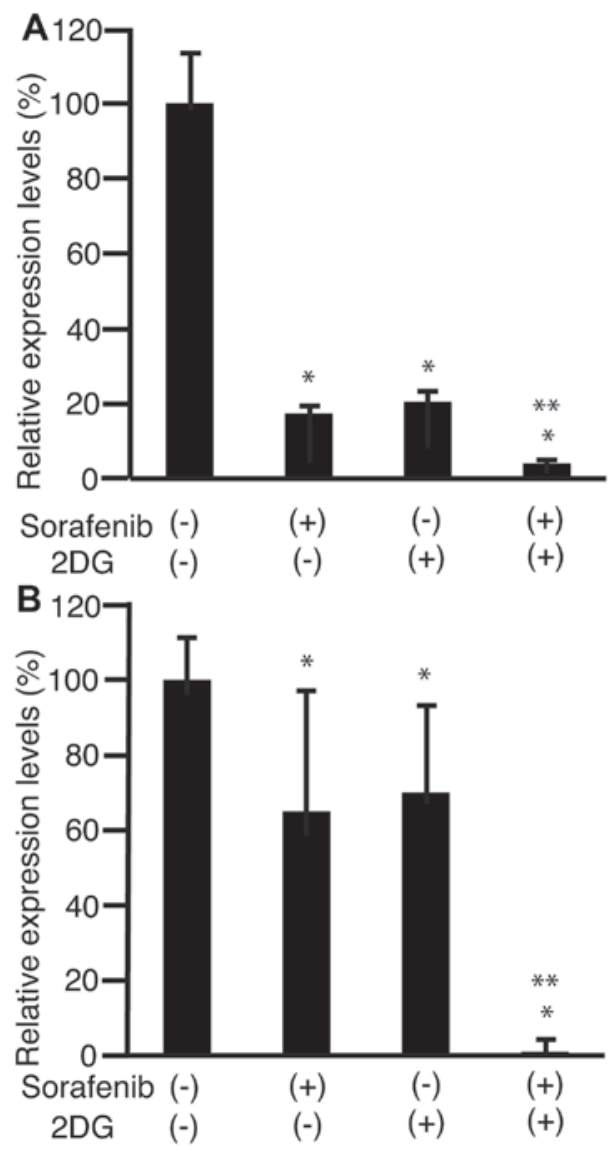

Figure 2. Levels of cyclin D1 expression. HLF cells (A) and PLC/PRF/5 cells (B) were cultivated with $3 \mu \mathrm{M}$ sorafenib, $1 \mu \mathrm{M} 2 \mathrm{DG}$, or a combination of the two. Cyclin D1 expression levels were compared with those of a control containing cells without sorafenib or 2DG. RNA was isolated and subjected to RT-qPCR to analyze cyclin D1 expression. ${ }^{*} \mathrm{P}<0.05$ against sorafenib (-) and 2DG (-) ${ }^{* *} \mathrm{P}<0.05$ against sorafenib (-) and $2 \mathrm{DG}(-)$, sorafenib $(-)$ and 2DG (+), and sorafenib (+) and 2DG (-), n=3. HLF cells, human liver fibroblast cells; PLC/PRF/5, the human hepatoma carcinoma cell line; 2DG, 2-deoxy-glucose; RT-qPCR, reverse transcription-quantitative polymerase chain reaction.

resistant to 2DG in these hypoxic regions (20). The combination of 2DG and sorafenib offers a promising strategy to overcome this resistance.

The feasibility of this approach is contingent on $2 \mathrm{DG}$ and sorafenib being safe for oral administration. A combination of 2DG and docetaxel as treatment for solid cancers has been 


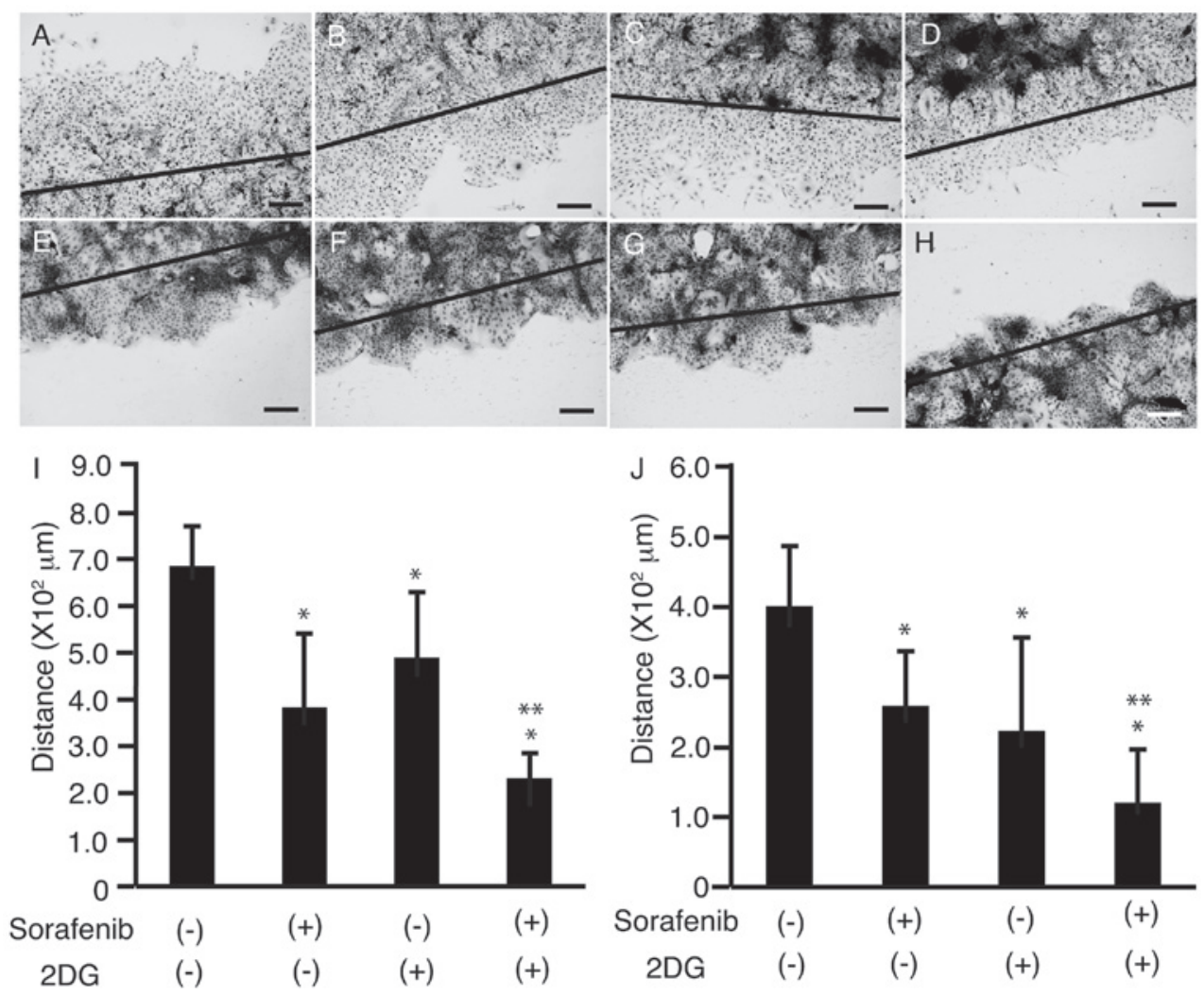

Figure 3. Scratch assay. HLF cells (A-D) and PLC/PRF/5 cells (E-H) in 4-well chamber slides were scratched with a sterile razor. Immediately after the scratch (solid line), no sorafenib or 2DG (A, E), $3 \mu \mathrm{M}$ sorafenib (B, F), $1 \mu \mathrm{M} 2 \mathrm{DG}(\mathrm{C}, \mathrm{G})$, or both (D, H) were added to the cells. Following $48 \mathrm{~h}$ in culture, the cells were stained with hematoxylin and eosin. Distance between the growing edge of the cell sheet and the scratched line was measured at five different points in HLF cells (I) and PLC/PRF/5 cells (J). Original magnification: x100, scale bar: $200 \mu \mathrm{M},{ }^{*} \mathrm{P}<0.05$ against sorafenib (-) and $2 \mathrm{DG}(-),{ }^{* *} \mathrm{P}<0.05$ against sorafenib $(-)$ and 2DG (-), sorafenib (-) and 2DG (+), and sorafenib (+) and 2DG (-), n=5. HLF, Human liver fibroblast cells; 2DG, 2-deoxyglucose; PLC/PRF/5 cells, human hepatoma carcinoma cell line.
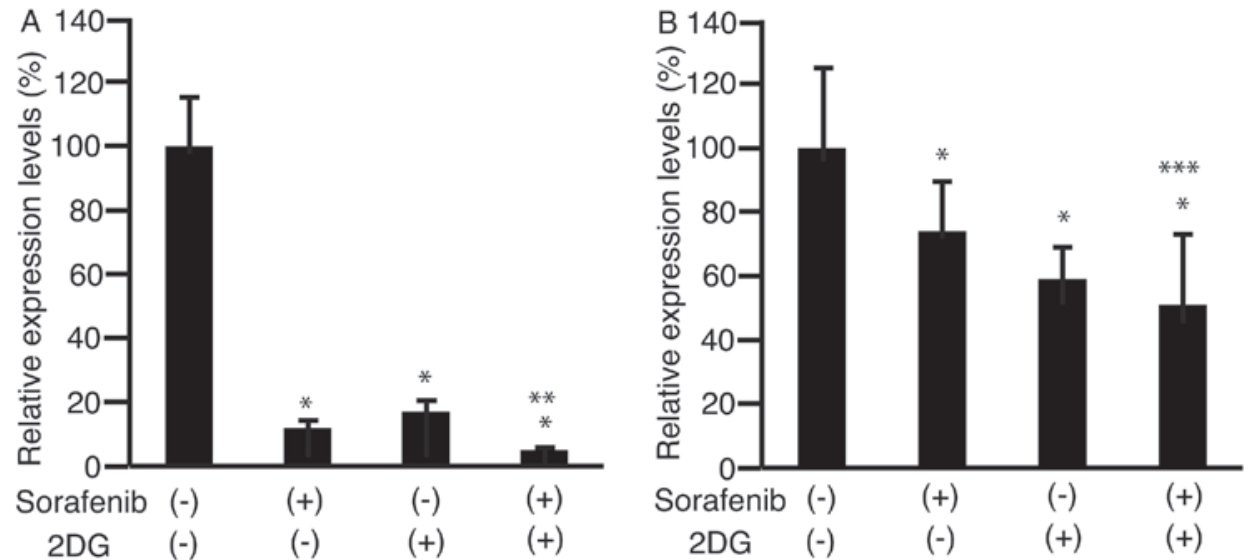

Figure 4. Expression levels of matrix metalloproteinase. HLF cells (A) and PLC/PRF/5 cells (B) were cultured in 6-well plates, and added to $3 \mu \mathrm{M}$ sorafenib $(+), 1 \mu \mathrm{M} 2 \mathrm{DG}(+)$, or a combination of both. Following $48 \mathrm{~h}$ in culture, RNA was isolated and subjected to RT-qPCR. Levels of matrix metalloproteinase expression were analyzed. "P<0.05 against sorafenib (-) and 2DG (-), ${ }^{* *} \mathrm{P}<0.05$ against sorafenib (-) and 2DG (-), sorafenib (-) and 2DG (+), and sorafenib (+) and 2DG (-), ${ }^{* * * *} \mathrm{P}<0.05$ against sorafenib (-) and 2DG (-), and sorafenib (-) and 2DG (+), n=3. HLF, Human liver fibroblast cells; 2DG, 2-deoxyglucose; PLC/PRF/5 cells, human hepatoma carcinoma cell line; RT-qPCR, reverse transcription-quantitative polymerase chain reaction.

investigated, and no serious adverse effects were reported (21). Furthermore, a phase I/II clinical trial investigating the combination of radiotherapy and 2DG against glioma reported no serious adverse events (22). These reports and the results of the current study suggest that the combination of 2DG and sorafenib is safe for oral administration, though further studies are required to confirm this.
The main limitation of the present study was the lack of in vivo data from xenograft animal models, therefore future studies are required that investigate the effects of systemic $2 \mathrm{DG}$ and sorafenib administration in vivo.

In conclusion, the current study demonstrated that 2DG and sorafenib suppressed the proliferation of HCC cells more effectively than sorafenib alone, and suppressed cell motility 
more effectively than 2DG or sorafenib alone. Therefore, combined treatment of 2DG and sorafenib may be an effective therapeutic strategy to treat cancer, including HCC.

\section{References}

1. Cameron AM: Screening for viral hepatitis and hepatocellular cancer. Surg Clin North Am 95: 1013-1021, 2015.

2. Khan FZ, Perumpail RB, Wong RJ and Ahmed A: Advances in hepatocellular carcinoma: Nonalcoholic steatohepatitis-related hepatocellular carcinoma. World J Hepatol 7 : 2155-2161, 2015.

3. Wells SA, Hinshaw JL, Lubner MG, Ziemlewicz TJ, Brace CL and Lee FT Jr: Liver ablation: Best practice. Radiol Clin North Am 53: 933-971, 2015.

4. Katano T, Mizoshita T, Senoo K, Sobue S, Takada H, Sakamoto T, Mochiduki H, Ozeki T, Kato A, Matsunami K, et al: The efficacy of transcatheter arterial embolization as the first-choice treatment after failure of endoscopic hemostasis and endoscopic treatment resistance factors. Dig Endosc 24: 364-369, 2012.

5. Furuse J, Ishii H, Nakachi K, Suzuki E, Shimizu S and Nakajima K: Phase I study of sorafenib in Japanese patients with hepatocellular carcinoma. Cancer Sci 99: 159-165, 2008.

6. Kuczynski EA, Lee CR, Man S, Chen E and Kerbel RS: Effects of sorafenib dose on acquired reversible resistance and toxicity in hepatocellular carcinoma. Cancer Res 75: 2510-2519, 2015.

7. Chen J, Jin R, Zhao J, Liu J, Ying H, Yan H, Zhou S, Liang Y, Huang D, Liang X, et al: Potential molecular, cellular and microenvironmental mechanism of sorafenib resistance in hepatocellular carcinoma. Cancer Lett 367: 1-11, 2015.

8. Vaitheesvaran B, Xu J, Yee J, Q-Y L, Go VL, Xiao GG and Lee WN: The Warburg effect: A balance of flux analysis. Metabolomics 11: 787-796, 2015.

9. Jang M, Kim SS and Lee J: Cancer cell metabolism: Implications for therapeutic targets. Exp Mol Med 45: e45, 2013.

10. Granja S, Pinheiro C, Reis RM, Martinho O and Baltazar F: Glucose addiction in cancer therapy: Advances and drawbacks. Curr Drug Metab 16: 221-242, 2015.

11. Zhang D, Li J, Wang F, Hu J, Wang S and Sun Y: 2-DeoxyD-glucose targeting of glucose metabolism in cancer cells as a potential therapy. Cancer Lett 355: 176-183, 2014.
12. Brito AF, Mendes M, Abrantes AM, Tralhão JG and Botelho MF: Positron emission tomography diagnostic imaging in multidrug-resistant hepatocellular carcinoma: Focus on 2-deoxy-2-(18F)Fluoro-D-Glucose. Mol Diagn Ther 18: 495-504, 2014.

13. Davies B and Fried M: The L19 ribosomal protein gene (RPL19): Gene organization, chromosomal mapping, and novel promoter region. Genomics 25: 372-380, 1995.

14. Vandooren J, Van den Steen PE and Opdenakker G: Biochemistry and molecular biology of gelatinase B or matrix metalloproteinase- 9 (MMP-9): The next decade. Crit Rev Biochem Mol Biol 48: 222-272, 2013.

15. Zhang Y, Shen Y, Cao B, Yan A and Ji H: Elevated expression levels of androgen receptors and matrix metalloproteinase-2 and -9 in 30 cases of hepatocellular carcinoma compared with adjacent tissues as predictors of cancer invasion and staging. Exp Ther Med 9: 905-908, 2015.

16. Wu J, Kou W, Gao MT, Zhou YN, Wang AQ, Xue QJ and Qiao L: Effects of \{2-[(3-carboxy-1-oxoprogyl)amino]-2-deoxy-D-glucose $\}$ on human hepatocellular carcinoma cell line. Acta Pharmacol Sin 26: 635-640, 2005 .

17. Tomizawa M, Kondo F and Kondo Y: Growth patterns and interstitial invasion of small hepatocellular carcinoma. Pathol Int 45: 352-358, 1995

18. Takemura A, Che XF, Tabuchi T, Moriya S, Miyazawa K and Tomoda A: Enhancement of cytotoxic and pro-apoptotic effects of 2-aminophenoxazine-3-one on the rat hepatocellular carcinoma cell line dRLh-84, the human hepatocellular carcinoma cell line HepG2, and the rat normal hepatocellular cell line RLN-10 in combination with 2-deoxy-D-glucose. Oncol Rep 27: 347-355, 2012.

19. Lv Y,Zhao S, Han J,Zheng L, Yang Z and Zhao L: Hypoxia-inducible factor-1 $\alpha$ induces multidrug resistance protein in colon cancer. Onco Targets Ther 8: 1941-1948, 2015.

20. Maher JC, Wangpaichitr M, Savaraj N, Kurtoglu M and Lampidis TJ: Hypoxia-inducible factor-1 confers resistance to the glycolytic inhibitor 2-deoxy-D-glucose. Mol Cancer Ther 6: 732-741, 2007.

21. Raez LE, Papadopoulos K, Ricart AD, Chiorean EG, Dipaola RS, Stein MN, Rocha Lima CM, Schlesselman JJ, Tolba K, Langmuir VK, et al: A phase I dose-escalation trial of 2-deoxy-D-glucose alone or combined with docetaxel in patients with advanced solid tumors. Cancer Chemother Pharmacol 71: 523-530, 2013.

22. Singh D, Banerji AK, Dwarakanath BS, Tripathi RP, Gupta JP, Mathew TL, Ravindranath T and Jain V: Optimizing cancer radiotherapy with 2-deoxy-d-glucose dose escalation studies in patients with glioblastoma multiforme. Strahlenther Onkol 181: 507-514, 2005 . 